Werner Bußmann u.a.

\title{
Geothermie -
}

Energie aus dem Innern der Erde

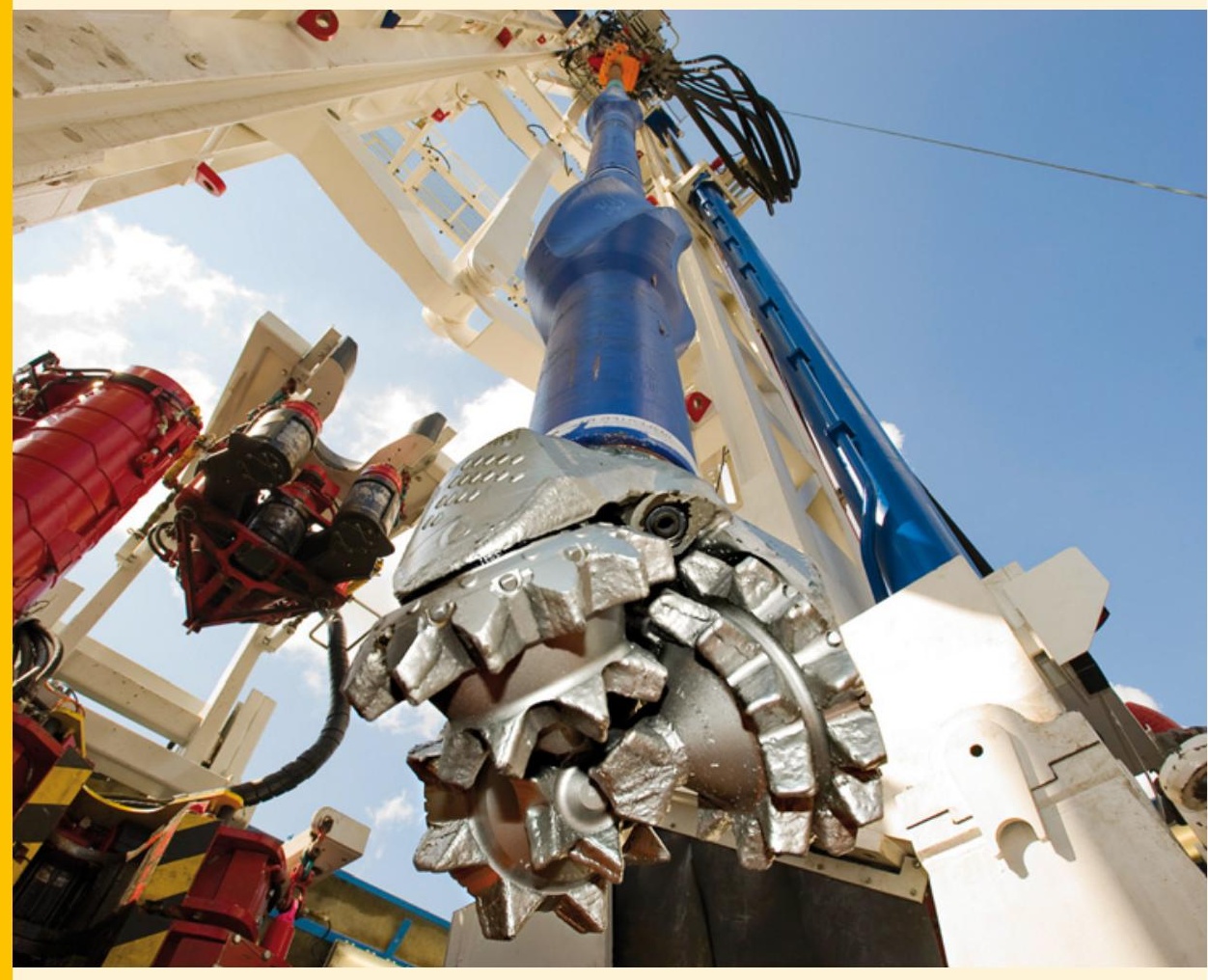

Fraunhofer IRB _Verlag 
BINE-Fachbuch

Werner Bußmann, Stephanie Frick, Ralf Fritschen, Ernst Huenges, Reinhard Jung, Frank Kabus, Martin Kaltschmitt, Oliver Kohlsch, Stefan Kranz, Inga Moeck, Horst Rüter, Ali Saadat, Angela Spalek, Helmut Tenzer, Günter Zimmermann

\section{Geothermie}


BINE-Fachbuch

\section{Geothermie}

Energie aus dem Innern der Erde

Die Autoren:

Werner Bußmann

Stephanie Frick

Ralf Fritschen

Ernst Huenges

Reinhard Jung

Frank Kabus

Martin Kaltschmitt

Oliver Kohlsch

Stefan Kranz

Inga Moeck

Horst Rüter

Ali Saadat

Angela Spalek

Helmut Tenzer

Günter Zimmermann 
BINE Informationsdienst berichtet über Themen der Energieforschung: Neue Materialien, Systeme und Komponenten, innovative Konzepte und Methoden. BINE-Leser werden so über Erfahrungen und Lerneffekte beim Einsatz neuer Technologien in der Praxis informiert. Denn erstklassige Informationen sind die Grundlage für richtungsweisende Entscheidungen, sei es bei der Planung energetisch optimierter Gebäude, der Effizienzsteigerung industrieller Prozesse oder bei der Integration erneuerbarer Energien in bestehende Systeme.

Weitere Informationen finden Sie unter: www.bine.info

Für weitere Fragen stehen Ihnen zur Verfügung:

Uwe Milles

BINE Informationsdienst, FIZ Karlsruhe, Büro Bonn

Kaiserstr. 185-197, 53113 Bonn

Tel. 0228923 79-0, E-Mail: bine@fiz-karlsruhe.de, www.bine.info

Bibliografische Information der Deutschen Nationalbibliothek

Die Deutsche Nationalbibliothek verzeichnet diese Publikation in der Deutschen Nationalbibliografie; detaillierte bibliografische Daten sind im Internet über http://dnb.d-nb.de abrufbar.

ISBN: 978-3-8167-8321-3

ISBN Printausgabe: 978-3-8167-8321-3 I ISBN E-Book: 978-3-8167-8363-3

Layout: Dietmar Zimmermann I Umschlaggestaltung: Martin Kjer I Herstellung: Katharina Kimmerle Satz: Mediendesign Späth, Birenbach I Druck: DZA Druckerei zu Altenburg GmbH, Altenburg

Für den Druck des Buches wurde chlor- und säurefreies Papier verwendet.

Alle Rechte vorbehalten

Dieses Werk ist einschließlich aller seiner Teile urheberrechtlich geschützt. Jede Verwertung, die über die engen Grenzen des Urheberrechtsgesetzes hinausgeht, ist ohne schriftliche Zustimmung des Fraunhofer IRB Verlages unzulässig und strafbar. Dies gilt insbesondere für Vervielfältigungen, Übersetzungen, Mikroverfilmungen sowie die Speicherung in elektronischen Systemen.

Die Wiedergabe von Warenbezeichnungen und Handelsnamen in diesem Buch berechtigt nicht zu der Annahme, dass solche Bezeichnungen im Sinne der Warenzeichen- und Markenschutz-Gesetzgebung als frei zu betrachten wären und deshalb von jedermann benutzt werden dürften.

Sollte in diesem Werk direkt oder indirekt auf Gesetze, Vorschriften oder Richtlinien (z. B. DIN, VDI, VDE) Bezug genommen oder aus ihnen zitiert werden, kann der Verlag keine Gewähr für Richtigkeit, Vollständigkeit oder Aktualität übernehmen. Es empfiehlt sich, gegebenenfalls für die eigenen Arbeiten die vollständigen Vorschriften oder Richtlinien in der jeweils gültigen Fassung hinzuzuziehen

Titelbild: BMU-Bilddatenbank

(c) by FIZ Karlsruhe

Verlag und Vertrieb:

Fraunhofer IRB Verlag

Fraunhofer-Informationszentrum

Raum und Bau IRB

Nobelstraße 12, 70569 Stuttgart

Telefon (0711) 970-2500

Telefax (0711) 970-2508

E-Mail: irb@irb.fraunhofer.de

http://www.baufachinformation.de

Hinweis zu den Abbildungen: Soweit nachfolgend keine anderen Quellen genannt werden, stammen die Abbildungen von den Autoren. 


\section{Inhaltsverzeichnis}

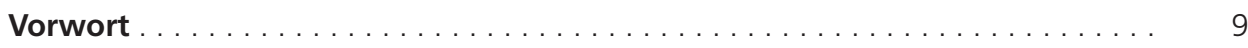

$1 \quad$ Ein Planet voller Energie $\ldots \ldots \ldots \ldots \ldots \ldots \ldots \ldots \ldots \ldots \ldots \ldots$

Werner Bußmann

2 Ideen - Innovationen - Technologien:

Es gibt viele Möglichkeiten, geothermische Energie zu nutzen . . . . . 16

Werner Bußmann

$2.1 \quad$ Weltweit ein Hoffnungsträger . . . . . . . . . . . . . . . . . . 19

2.2 Den Vulkan anzapfen: heißer Dampf und heißes Wasser . . . . . . . . . . . 23

2.3 Geothermische Potenziale in Deutschland . . . . . . . . . . . . . . . 26

3 Geologische Rahmenbedingungen und untertägige Erschließung . . . . . 35

Helmut Tenzer

3.1 Generelle Abfolge der Vorerkundung und Erschließungsarbeiten . . . . . . . . . 36

3.2 Durchführung einer Machbarkeitsstudie und geologische Vorerkundung . . . . . 38

3.3 Geophysikalische Vorerkundung ...................... 39

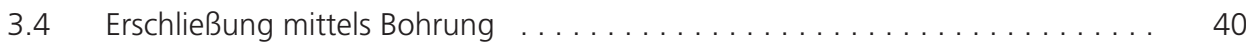

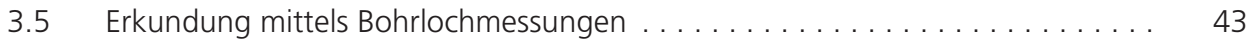

3.6 Hydraulischer Anschluss der Bohrung an das Reservoir . . . . . . . . . . . . 46

3.7 Erschließung über Doubletten und Tripletten . . . . . . . . . . . . . . . 47

3.8 Innovative Erschließungsmethoden tiefer heißer Gesteine . . . . . . . . . . . . . . 49

$4 \quad$ Hydrothermale Energie - Wärme und Strom aus Thermalwasser . . . . 51 Werner Bußmann

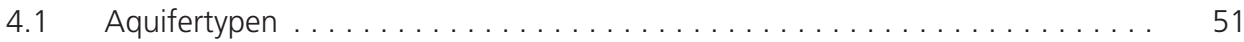

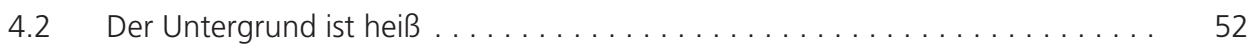

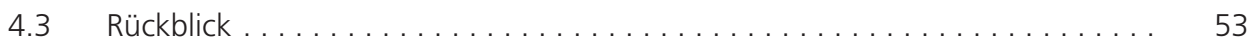

4.4 Thermalwasserregionen in Deutschland . . . . . . . . . . . . . . . 55

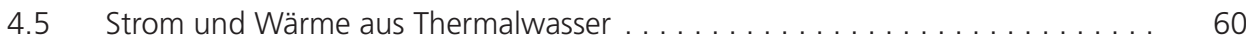

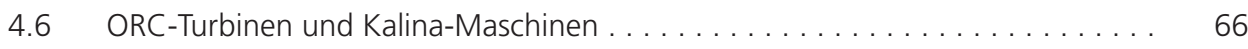

4.7 Hydrothermale Geothermie und Fernwärme: Konkurrenzfähig? Frank Kabus . . 68

4.8 Geotls .............................. 78

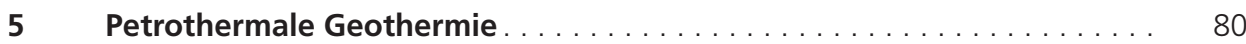

Reinhard Jung, Helmut Tenzer

$5.1 \quad$ Petrothermale Ressourcen in Deutschland . . . . . . . . . . . . . . . 80

5.1 .1 Geologie-Tektonik . . . . . . . . . . . . . . . . . . . 83

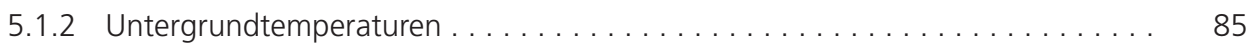


5.1 .3 Gebirgsspannungen und Seismizität . . . . . . . . . . . . . . . 86

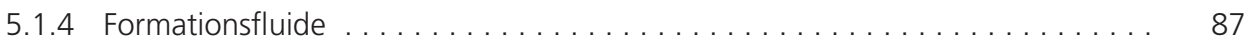

5.2 Erschließungstechnik und Forschungsprojekte . . . . . . . . . . . . 88

5.2.1 Schlüsseltechniken: Risserzeugung, seismische Rissortung und Richtbohrverfahren ... . . . . . . . . . . . . . . . . . . . . . . . 89

5.2 .2 Projekte . . . . . . . . . . . . . . . . . . . . . . . . . . . . . . 90

$5.3 \quad$ Installation eines petrothermalen Systems $\ldots \ldots \ldots \ldots \ldots \ldots \ldots \ldots \ldots$

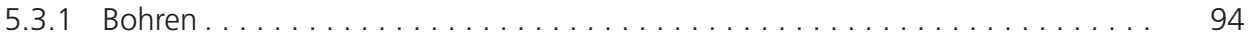

5.3 .2 Bohrlochmessungen............................ 95

5.3.3 Schaffung des untertägigen Wärmetauschers . . . . . . . . . . . . . . 96

5.3.4 Untersuchung des untertägigen Wärmetauschers . . . . . . . . . . . . . . . 97

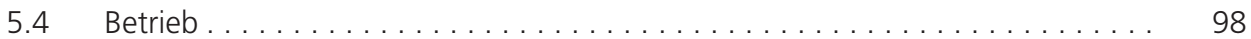

5.5 Anlagenbeispiel - Europäisches Forschungsprojekt Soultz-sous-Forêts $\ldots \ldots \ldots 100$

$6 \quad$ Umweltbilanz tiefer Geothermie . . . . . . . . . . . . . . . . . . . . 105 Horst Rüter, Martin Kaltschmitt, Stephanie Frick

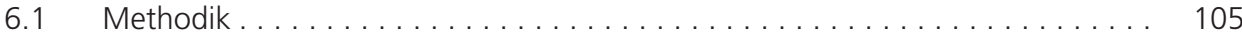

6.2 Untersuchte Referenzanlagen . . . . . . . . . . . . . . . . . . 106

6.3 Bilanzergebnisse . . . . . . . . . . . . . . . . . . . . . 108

$7 \quad$ Exkurs Seismizität . . . . . . . . . . . . . . . . . . . . . . . . . . . . . . . . . 114 Horst Rüter, Ralf Fritschen

$7.1 \quad$ Erdbeben - Skalen und Begriffe . . . . . . . . . . . . . . . . 115

7.2 Natürliche Seismizität, Statistik der Erdbeben, Makroseismizität, Erdbebenzonen, Spannungsfeld der Erdkruste . . . . . . . . . . . . . . . . . . 116

7.3 Induzierte Seismizität . . . . . . . . . . . . . . . . . . . . . . . . . . . . . . . . . . 120

7.4 Induzierte Seismizität in der Geothermie . . . . . . . . . . . . . . . . . . . . . . . . 122

7.5 Zusammenhang mit Betriebsparametern . . . . . . . . . . . . . . . . . . . 123

7.6 Schadenswirkung und seismisches Risiko . . . . . . . . . . . . . . . . 123

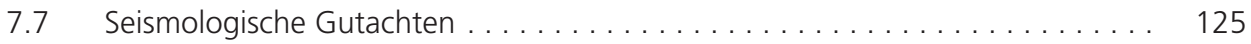

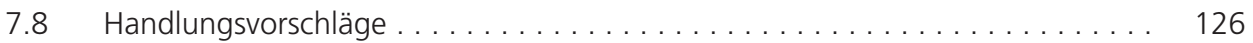

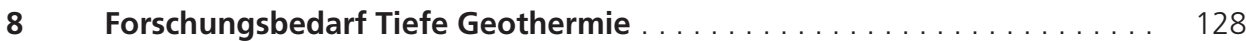

Ernst Huenges, Inga Moeck, Günter Zimmermann, Ali Saadat,

Stephanie Frick, Stefan Kranz und Angela Spalek

8.1 Ziele der Forschung . . . . . . . . . . . . . . . . . . . . . . . . . . . . . . 129

8.2 Fündigkeit, Reservoirgestaltung, Systemverlässlichkeit - Hauptkriterien auf dem Weg zur Wirtschaftlichkeit geothermischer Systeme . . . . . . . . . . . . . . . 129

8.3 Effiziente Systemlösungen Von der Erkundung des Reservoirs bis zur Energiewandlung im Kraftwerk . . . . 131

8.3.1 Fündigkeitsprognose: Für oder Wider ein Projekt . . . . . . . . . . . . . . 131 
8.3.2 Reservoirengineering - Schlüssel zur Wirtschaftlichkeit . . . . . . . . . . . 133

8.3.3 Systemverlässlichkeit für einen nachhaltigen Anlagenbetrieb . . . . . . . . . . . 134

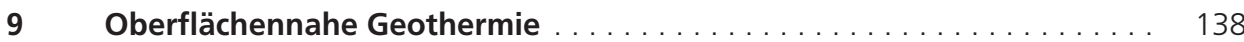
Oliver Kohlsch

9.1 Allgemeine Systembeschreibung oberflächennaher Geothermiesysteme . . . . 138

9.2 Erdwärmesonden . . . . . . . . . . . . . . . . . . . . . . . . . . . . . . . . . . . 139

9.2.1 Heizbetrieb . . . . . . . . . . . . . . . . . . . . . . . . . . . . 142

9.2 .2 Heiz- und Kühlbetrieb . . . . . . . . . . . . . . . . . . . . . . . . 143

9.2.3 Dimensionierung einer Erdwärmesondenanlage . . . . . . . . . . . . . . . . . . 144

9.3 Erdwärmekollektoren . . . . . . . . . . . . . . . . . . . . . . . . . . . . . . . . 147

9.4 Brunnenanlagen . . . . . . . . . . . . . . . . . . . . . . . . . . . . 150

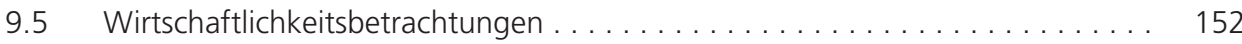

10 Zitierte und sonstige verwendete Literatur, Abbildungsverzeichnis . . . 153

10.1 Zitierte Literatur . . . . . . . . . . . . . . . . . . . . . . . . . . . . . . . . . . 153

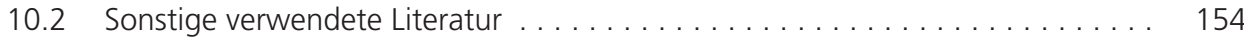

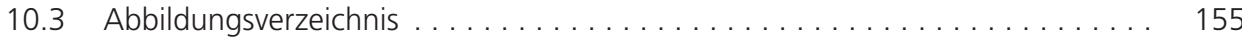

11 Laufende und abgeschlossene Forschungsvorhaben aus der Energieforschung der Bundesregierung . . . . . . . . . . . . . . . . 157

11.1 Laufende und kürzlich abgeschlossene Forschungsvorhaben . . . . . . . . . 157

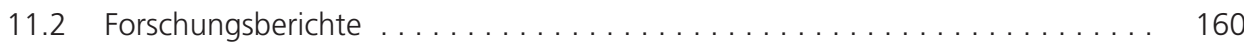

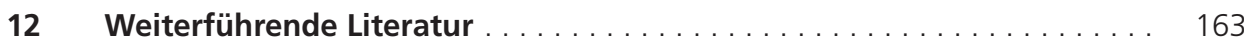

12.1 Literatur . . . . . . . . . . . . . . . . . . . . . . . . . . . . . 163

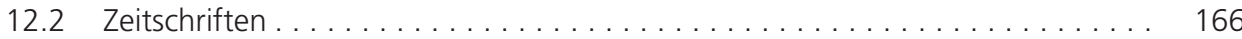

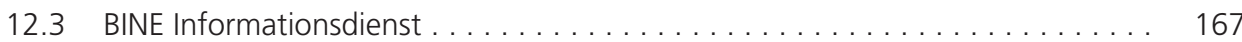

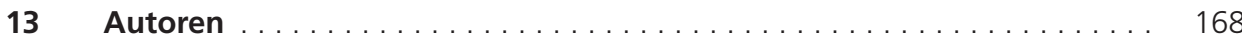




\section{Vorwort}

Die meisten erneuerbaren Energietechnologien nutzen direkt (Solarthermie, Photovoltaik und passive Systeme) oder indirekt (Windenergie, Wasserkraft, Biomassenutzung) die Sonnenenergie. Mit der Wärme der Erde, der Geothermie, existiert eine zweite erneuerbare Energiequelle unabhängig von der Sonne. Diese Wärme unter unseren Füßen kann zur Wärmeversorgung von Gebäuden und Siedlungen sowie für die geothermische Stromerzeugung genutzt werden. Ihr Vorteil: Sie steht unabhängig von Tages- und Jahreszeiten zur Verfügung.

In Deutschland spricht man von tiefer Geothermie, wenn Bohrungen geothermische Energie in einer Tiefe von über $400 \mathrm{~m}$ und einer Temperatur von über $20^{\circ} \mathrm{C}$ erschließen. Die tiefe Geothermie nutzt entweder natürliche Warmwasservorkommen (hydrothermale Anlagen) oder die im Gestein gespeicherte Wärme (petrothermale Anlagen). Beide können zur Wärmeversorgung und zur Stromerzeugung eingesetzt werden. Bislang sind in Deutschland (Stand: 2010) 17 größere Geothermieanlagen in Betrieb, darunter 4 Kraftwerke. Für 150 weitere geplante Projekte wurde eine bergrechtliche Aufsuchungserlaubnis erteilt.

Deutschland verfügt über weitaus mehr geothermische Ressourcen als bisher genutzt werden. Dieses BINE-Fachbuch stellt die geologischen Grundlagen und die Erschließungstechnologien vor, gibt einen Überblick über die hydrothermalen und petrothermalen Anlagenkonzepte und diskutiert die Umweltbilanz. Weitere Themen sind die aktuellen Forschungsziele und ein knapper Überblick über die oberflächennahe Geothermie. Diese wird in den BINE-Fachbüchern »Erdwärme für Bürogebäude nutzen« und "Wärmepumpen - Heizen mit Umweltenergie« ausführlicher vorgestellt. Beide Formen der Erdwärmenutzung werden in der künftigen Energieversorgung Deutschlands einen wachsenden Beitrag leisten.

FIZ Karlsruhe

BINE Informationsdienst 
